\title{
Hemopoietic Colony-forming Cells in Umbilical Cord Blood with Extensive Capability to Generate Mono- and Multipotential Hemopoietic Progenitors
}

\author{
Tatsutoshi Nakahata and Makio Ogawa, Department of Medicine, Medical \\ University of South Carolina, Charleston, South Carolina 29425; Veterans \\ Administration Medical Center, Charleston, South Carolina 29403
}

\begin{abstract}
A B S T R A C T We report identification of a unique class of human hemopoietic colony-forming cells with extensive ability to generate progenitors for secondary colonies. Mononuclear cells isolated from human umbilical cord blood formed colonies consisting of 40-500 blast cells after $25 \mathrm{~d}$ of incubation in methylcellulose culture in the presence of erythropoietin and medium conditioned by phytohemagglutinin-stimulated leukocytes. Replating of these blast cell colonies revealed that $100 \%$ of the primary colonies had the ability to generate secondary colonies, including multipotential colonies. These colonies could be distinguished from other hemopoietic colonies in situ by the complete absence of signs of terminal differentiation. Replating of granulocyte-erythrocyte-macrophage-megakaryocyte (GEMM) colonies, consisting of an average of 2 $\times 10^{4}$ cells, revealed less capacity for secondary colony formation. This human blast cell colony assay may provide a method for quantitation of more primitive hemopoietic stem cells than progenitors for GEMM colonies (CFU-GEMM) in man.
\end{abstract}

\section{INTRODUCTION}

An in vitro assay system for pluripotent hemopoietic stem cells that possess the capacity to self-renew and provide differentiated progenies has been pursued by investigators for several years. Recently, Johnson and Metcalf (1) and our group (2) described mouse hemopoietic progenitors that are capable of differentiation in more than three cell lineages in culture. Subsequently, human multipotential hemopoietic progenitors that show differentiation in granulocyte-

\footnotetext{
Address reprint requests to Dr. Ogawa, VA Medical Center.

Received for publication 2 June 1982 and in revised form 23 August 1982.
}

erythrocyte-macrophage-megakaryocyte (GEMM) ${ }^{1}$ lineages were identified in cell culture (3). Detailed studies of the potentials for self-renewal and secondary colony formation have been performed using these large multilineage colonies (4-6).

We recently identified a class of mouse hemopoietic colony-forming units (CFU) that appear to be more primitive than CFU-GEMM in the hierarchy of stem cells (7). The colonies consisted of $<1,000$ blast cells and could be distinguished from other hemopoietic colonies in situ by the complete absence of signs of terminal differentiation. Replating of these colonies revealed their extensive self-renewal capacity and ability to generate secondary colonies, many of which were multipotential in nature. Comparison of the progenitor incidences between the stem cell colonies and GEMM colonies indicated that the progenitors for the stem cell colonies are more primitive than CFUGEMM. In this communication, we report our observations on human hemopoietic colonies consisting of only blast cells, many of which are hemopoietic progenitors.

\section{METHODS}

Clonal cell culture. Mononuclear cells isolated from umbilical cord blood samples were cultured at a concentration of $2 \times 10^{4}$ cells $/ \mathrm{ml}$ by using a modification (8) of the methylcellulose culture described by Iscove et al. (9). We used $2 \mathrm{U}$ of partially-purified human urinary erythropoietin with a specific activity of $840 \mathrm{U} / \mathrm{mg}$ protein (kindly provided by Dr. Makoto Kawakita, Kumamoto University, Kumamoto, Japan) and 5\% (vol/vol) medium conditioned by phytohemagglutinin-stimulated leukocytes. Dishes were incubated at $37^{\circ} \mathrm{C}$ in a humidified atmosphere flushed with $5 \% \mathrm{CO}_{2}$ in air. Megakaryocyte colonies were scored on day 10, blast cell colonies on day 25, and all other colonies on day 16 of

\footnotetext{
${ }^{1}$ Abbreviations used in this paper: CFU, colony-forming units; GEMM, granulocyte-erythrocyte-macrophage-megakaryocyte; GM, granulocyte-macrophage.
} 
culture. Megakaryocyte colonies were identified using the criteria described previously (10). Colonies considered to contain two or more cell lineages were individually lifted from the dishes, centrifuged onto slide glasses by use of a cytocentrifuge, and stained with May-Grunwald Giemsa for determination of cellular compositions. Characterization of granulocyte-macrophage-megakaryocyte colonies and erythro-eosinophil colonies has been presented previously $(8,11)$.

Replating experiments. On day 16 and day 25 of culture respectively, individual GEMM colonies and individual blast cell colonies, which were clearly isolated from other colonies, were lifted from the methylcellulose medium using a $3-\mu 1$ Eppendorf pipette under direct microscopic visualization and were suspended in $0.2 \mathrm{ml} \alpha$-medium. After gentle pi- petting, samples were divided into two aliquots; one-half to be processed for morphologic examination of constituent cells and the other half to be added to culture dishes containing $0.9 \mathrm{ml}$ methylcellulose culture medium with conditioned medium and erythropoietin. The size of individual blast cell colonies was determined by direct cell counting in situ. The size of GEMM colonies was determined by counting in hemocytometer chambers and on the cytocentrifuge preparations as we described previously (8).

\section{RESULTS}

Colony formation. When dishes were incubated for $16 \mathrm{~d}$, most of the colonies revealed signs of terminal
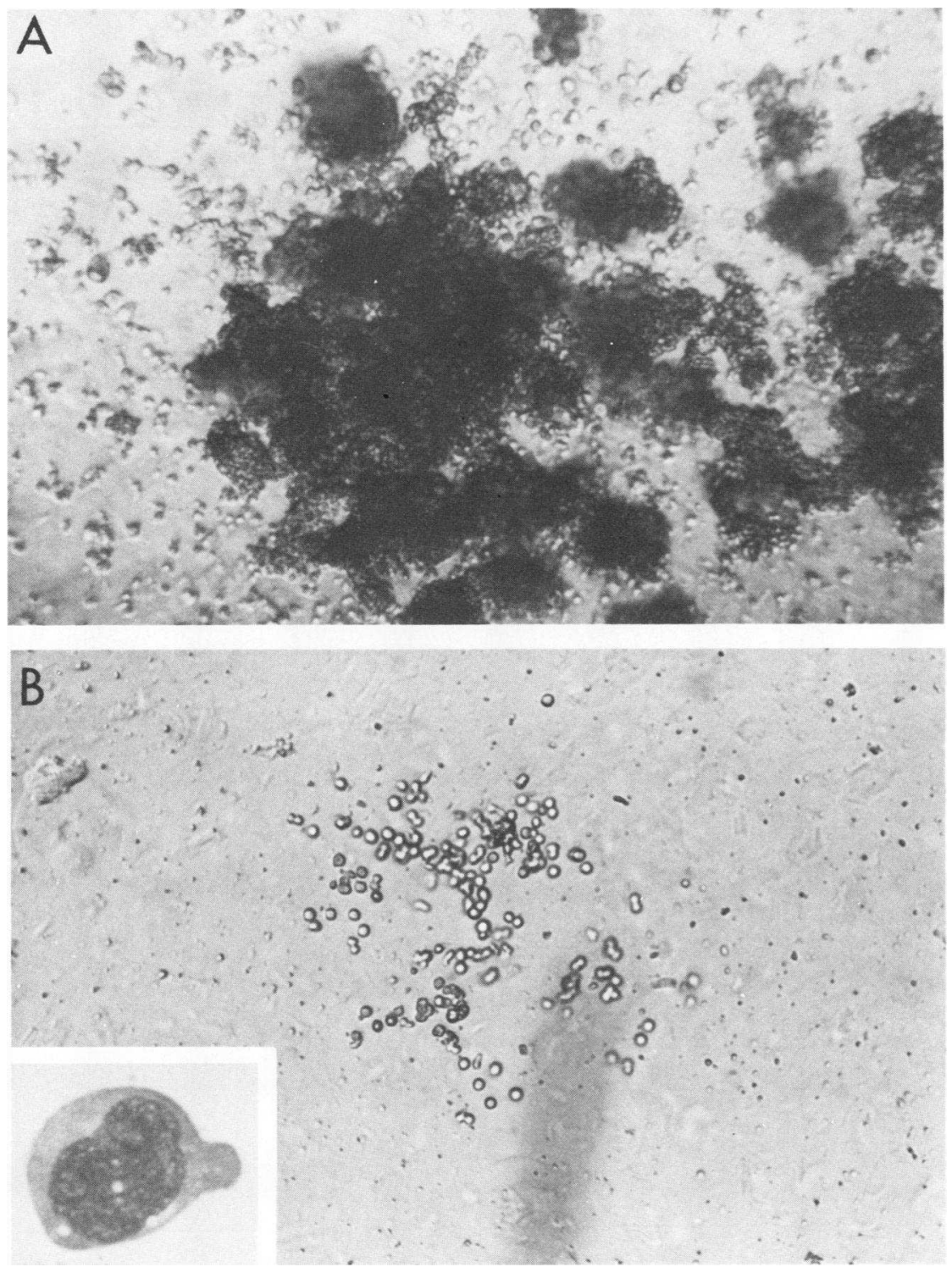

Figure 1A Day 16 macroscopic GEMM colony $(\times 70)$. B Day 25 stem cell colony consisting of cells with no signs of terminal differentiation $(\times 120)$; inset, a representative blast cell revealing immature nucleus and absence of cytoplasmic differentiation $(\times 2,000)$. 
differentiation; i.e., the red color of hemoglobin, large megakaryocytes and neutrophilic granulocytes recognizable by their polygonal shape. A large GEMM colony seen on day 16 of culture is shown in Fig. 1A. When culture was continued beyond 16 days, some colonies completely disintegrated and almost all colonies showed signs of cell degeneration. On day 25 of culture, only four types of colonies were recognizable; partly degenerated eosinophil colonies, macrophage colonies, megakaryocyte colonies consisting of $>50$ megakaryocytes (10), and colonies consisting of 40 500 loosely arranged, round cells with no signs of terminal differentiation (Fig. 1B). The appearance of the latter colonies was identical to that of mouse stem cell colonies we previously described (7). When these colonies were stained with May-Grunwald Giemsa, only blast cells were seen (Fig. 1B inset). These cells were negative with benzidine, myeloperoxidase, Luxol-fast blue and alcian-blue-safranin staining. The incidence of the human blast cell colonies was $0.9 \pm 0.4$ per 2 $\times 10^{4}$ mononuclear cord blood cells.

Replating experiments. The ability of the human blast cell colonies to generate a large number of secondary colonies including mixed hemopoietic colonies was determined by replating experiments. The results of replating of a total of 30 blast cell colonies are presented in Table I. All colonies contained significant numbers of granulocyte-macrophage CFU (CFU-GM).

TABLE I

Results of Replating Experiments of Blast Cell Colonies

\begin{tabular}{|c|c|c|c|c|c|c|c|c|c|c|c|c|c|c|}
\hline \multirow{2}{*}{$\begin{array}{l}\text { Cord } \\
\text { blood } \\
\text { sample }\end{array}$} & \multirow{2}{*}{$\begin{array}{l}\text { Colony } \\
\text { number }\end{array}$} & \multirow{2}{*}{$\begin{array}{c}\text { Total } \\
\text { cell } \\
\text { count }\end{array}$} & \multicolumn{11}{|c|}{ Number of secondary colonies ${ }^{\circ}$ per $1 / 2$ primary colony } & \multirow{2}{*}{$\begin{array}{l}\text { Total } \\
\text { replating } \\
\text { efficiency }\end{array}$} \\
\hline & & & Blast & B & GM & M & Eo & GEM & EM & EEo & GMM & GEMM & Total & \\
\hline & & & & & & & & & & & & & & $\%$ \\
\hline A & 1 & 300 & 2 & 32 & 26 & 7 & 2 & 2 & 5 & 1 & 2 & 1 & 80 & 53 \\
\hline \multirow[t]{5}{*}{ B } & 2 & 54 & 0 & 1 & 4 & 1 & 0 & 2 & 0 & 0 & 0 & 0 & 8 & 30 \\
\hline & 3 & 202 & 0 & 5 & 7 & 3 & 0 & 2 & 1 & 0 & 3 & 1 & 22 & 22 \\
\hline & 4 & 81 & 0 & 0 & 2 & 1 & 0 & 0 & 0 & 0 & 1 & 0 & 4 & 10 \\
\hline & 5 & 108 & 0 & 0 & 2 & 1 & 0 & 1 & 0 & 0 & 0 & 0 & 4 & 7 \\
\hline & 6 & 201 & 0 & 1 & 5 & 4 & 0 & 1 & 0 & 0 & 0 & 1 & 12 & 12 \\
\hline \multirow[t]{5}{*}{ C } & 7 & 41 & 0 & 1 & 2 & 2 & 0 & 1 & 0 & 0 & 0 & 0 & 6 & 29 \\
\hline & 8 & 177 & 0 & 0 & 2 & 1 & 0 & 0 & 0 & 0 & 0 & 0 & 3 & 3 \\
\hline & 9 & 51 & 0 & 0 & 2 & 1 & 0 & 0 & 0 & 0 & 1 & 0 & 4 & 16 \\
\hline & 10 & 245 & 0 & 10 & 11 & 3 & 0 & 1 & 2 & 0 & 1 & 3 & 31 & 25 \\
\hline & 11 & 129 & 0 & 0 & 2 & 2 & 0 & 0 & 0 & 0 & 0 & 0 & 4 & 6 \\
\hline \multirow[t]{6}{*}{ D } & 12 & 382 & 0 & 0 & 6 & 2 & 0 & 0 & 0 & 0 & 0 & 0 & 8 & 4 \\
\hline & 13 & 142 & 0 & 0 & 10 & 3 & 0 & 0 & 0 & 0 & 0 & 0 & 13 & 18 \\
\hline & 14 & 163 & 0 & 1 & 3 & 3 & 0 & 1 & 0 & 0 & 0 & 0 & 8 & 10 \\
\hline & 15 & 92 & 0 & 0 & 4 & 3 & 0 & 0 & 0 & 0 & 1 & 0 & 8 & 17 \\
\hline & 16 & 246 & 0 & 0 & 6 & 3 & 0 & 0 & 0 & 0 & 3 & 0 & 12 & 10 \\
\hline & 17 & 123 & 0 & 0 & 4 & 4 & 0 & 0 & 0 & 0 & 1 & 4 & 13 & 21 \\
\hline \multirow[t]{9}{*}{$\mathrm{E}$} & 18 & 42 & 0 & 1 & 2 & 1 & 0 & 1 & 0 & 0 & 0 & 1 & 6 & 29 \\
\hline & 19 & 222 & 0 & 0 & 8 & 10 & 0 & 1 & 0 & 2 & 0 & 1 & 22 & 20 \\
\hline & 20 & 253 & 1 & 1 & 7 & 1 & 0 & 0 & 0 & 0 & 0 & 0 & 11 & 9 \\
\hline & 21 & 118 & 0 & 1 & 8 & 1 & 1 & 0 & 0 & 0 & 0 & 0 & 11 & 19 \\
\hline & 22 & 197 & 0 & 5 & 10 & 10 & 0 & 0 & 0 & 1 & 3 & 1 & 30 & 30 \\
\hline & 23 & 79 & 0 & 0 & 7 & 1 & 0 & 0 & 0 & 0 & 0 & 0 & 8 & 20 \\
\hline & 24 & 198 & 0 & 0 & 7 & 2 & 0 & 0 & 0 & 0 & 0 & 0 & 9 & 9 \\
\hline & 25 & 102 & 0 & 2 & 5 & 1 & 0 & 1 & 0 & 0 & 0 & 1 & 10 & 20 \\
\hline & 26 & 500 & 0 & 2 & 31 & 10 & 0 & 3 & 1 & 0 & 5 & 5 & 57 & 23 \\
\hline \multirow[t]{5}{*}{$\mathbf{F}$} & 27 & 128 & 0 & 1 & 10 & 3 & 0 & 1 & 0 & 0 & 0 & 0 & 15 & 23 \\
\hline & 28 & 98 & 0 & 0 & 4 & 1 & 0 & 1 & 0 & 0 & 1 & 1 & 8 & 16 \\
\hline & 29 & 256 & 0 & 4 & 11 & 2 & 0 & 1 & 0 & 0 & 2 & 4 & 24 & 19 \\
\hline & 30 & 108 & 0 & 0 & 3 & 0 & 0 & 0 & 0 & 0 & 1 & 0 & 4 & 7 \\
\hline & & 168 & & & & & & & & & & & \multicolumn{2}{|c|}{ Mean 18} \\
\hline
\end{tabular}

- Blast, blast cell colonies; B, erythroid bursts; GM, colonies containing neutrophilic granulocytes and/or macrophages; M, megakaryocyte colonies; Eo, eosinophil colonies; GEM, colonies containing erythrocytes, neutrophilic granulocytes and/or macrophages; EM, colonies containing erythrocytes and megakaryocytes; EEo, colonies containing erythrocytes and eosinophils; GMM, colonies containing macrophages, megakaryocytes, and/or granulocytes. 
12 of the 30 blast cell colonies produced GEMM colonies. An average of $18 \%$ of the constituent cells of individual blast cell colonies proved to be hemopoietic progenitors. Varying combinations of differentiation capabilities were observed. However, we were unable to detect lymphoid colonies in the replating experiments. Two blast cell colonies produced one or two colonies consisting of only blast cells. The morphology of these colonies was confirmed by staining, but tertiary culture resulted in extremely poor growth of colonies. For comparison, we replated 25 GEMM colonies that were present in the same culture dishes as groups $\mathrm{E}$ and $\mathrm{F}$ in Table $\mathrm{I}$. The results are presented in Table II. Despite the large number of constituent cells in the GEMM colonies, the incidences of hemopoietic progenitors were significantly lower than those presented in Table I. For example, not all of the GEMM colonies produced secondary GM colonies, and the number of CFU-GEMM in individual GEMM colonies were clearly fewer than those in blast cell colonies.

\section{DISCUSSION}

In this report, we have presented evidence for the presence, in human umbilical cord blood, of progen- itors for the colonies that resemble murine stem cell colonies. The notion that these progenitors are more primitive in the stem cell hierarchy than CFU-GEMM was suggested by the following observations: $(a)$ cells constituting the blast cell colonies possess slower proliferative rates than those in GEMM colonies, since only an average of 168 cells were produced in the blast cell colonies after $25 \mathrm{~d}$ of culture. GEMM colonies attained an average size of $2.0 \times 10^{4}$ cells after $16 \mathrm{~d}$ of incubation. This slow proliferative rate of cells in the blast cell colonies is in agreement with the general concept in hemopoiesis that the primitive stem cells are quiescent. (b) Whereas cells in GEMM colonies revealed the signs of terminal differentiation after 16 $\mathrm{d}$ of culture, those in the blast cell colonies showed no differentiation even after longer incubation $(c)$ The replating of the blast cell colonies revealed a higher capacity for producing secondary colonies than GEMM colonies.

We did not identify lymphoid colonies in the replating experiments of blast cell colonies. Recently, Messner et al. (12) reported the presence of T lymphocytes in human mixed hemopoietic colonies grown in the presence of $\mathrm{T}$ lymphocyte conditioned medium.

TABLE II

Results of Replating Experiments of GEMM Colonies

\begin{tabular}{|c|c|c|c|c|c|c|c|c|c|c|c|c|}
\hline \multirow{2}{*}{$\begin{array}{l}\text { Cord } \\
\text { blood } \\
\text { sample }\end{array}$} & \multirow{2}{*}{$\begin{array}{l}\text { Colony } \\
\text { number }\end{array}$} & \multirow{2}{*}{$\begin{array}{c}\text { Total cell } \\
\text { count } \\
\left(\times 10^{4}\right)\end{array}$} & \multicolumn{10}{|c|}{ Number of secondary colonies ${ }^{\bullet}$ per $1 / 2$ primary colony } \\
\hline & & & Blast & B & GM & $\mathbf{M}$ & Eo & GEM & EM & EEo & GMM & GEMM \\
\hline \multirow[t]{13}{*}{$\mathrm{E}$} & 1 & 1.00 & 0 & 0 & 1 & 0 & 0 & 0 & 0 & $\mathbf{0}$ & 0 & 0 \\
\hline & 2 & 1.08 & 0 & 0 & 3 & 0 & 0 & 0 & 0 & 0 & $\mathbf{0}$ & 0 \\
\hline & 3 & 2.12 & 0 & 6 & 5 & 1 & 4 & 1 & 1 & 0 & 0 & 1 \\
\hline & 4 & 3.32 & 0 & 0 & 1 & 0 & 0 & 0 & 0 & 0 & 0 & 0 \\
\hline & 5 & 0.98 & 0 & 2 & 1 & 0 & 0 & 0 & 0 & 0 & 0 & 0 \\
\hline & 6 & 1.82 & 0 & 2 & 8 & 0 & 0 & 1 & 0 & 0 & 0 & 0 \\
\hline & 7 & 4.32 & 0 & 0 & 4 & 0 & 0 & 0 & 0 & 0 & 0 & 0 \\
\hline & 8 & 0.82 & 0 & 0 & 11 & 2 & 0 & 0 & 0 & 0 & 0 & 0 \\
\hline & 9 & 1.86 & 0 & 0 & 1 & 0 & 0 & 0 & 0 & 0 & 0 & 0 \\
\hline & 10 & 3.18 & 0 & 0 & 3 & 1 & 0 & 0 & 0 & 0 & 0 & 0 \\
\hline & 11 & 2.30 & 0 & 0 & 0 & 0 & 0 & 0 & 0 & 0 & 0 & 0 \\
\hline & 12 & 0.87 & 0 & 0 & 4 & 0 & 0 & 0 & 0 & 0 & 0 & 0 \\
\hline & 13 & 1.59 & 0 & 0 & 1 & 0 & 0 & 0 & 0 & 0 & 0 & 0 \\
\hline \multirow[t]{12}{*}{$\mathrm{F}$} & 14 & 3.68 & 0 & 0 & 0 & 0 & 0 & 0 & 0 & 0 & 0 & 0 \\
\hline & 15 & 2.42 & 0 & 0 & 2 & 0 & 1 & 0 & 0 & 0 & 0 & 0 \\
\hline & 16 & 1.01 & 0 & 0 & 0 & 0 & 0 & 0 & 0 & 0 & 0 & 0 \\
\hline & 17 & 1.98 & 0 & 0 & 4 & 0 & 0 & 0 & 0 & 0 & 0 & 0 \\
\hline & 18 & 0.72 & 0 & 0 & 0 & 0 & 0 & 0 & 0 & 0 & 0 & 0 \\
\hline & 19 & 0.59 & 0 & 1 & 11 & 2 & 0 & 1 & 0 & 0 & 0 & 0 \\
\hline & 20 & 1.97 & 0 & 0 & 1 & 0 & 0 & 0 & 0 & 0 & 0 & 0 \\
\hline & 21 & 2.22 & 0 & 0 & 3 & 1 & 0 & 0 & 0 & 0 & 0 & 0 \\
\hline & 22 & 0.79 & 0 & 0 & 4 & 0 & 0 & 0 & 0 & 0 & 0 & 0 \\
\hline & 23 & 1.83 & 0 & 0 & 1 & 0 & 0 & 0 & 0 & 0 & 0 & 0 \\
\hline & 24 & 4.68 & 0 & 2 & 15 & 4 & 0 & 2 & 1 & 0 & 1 & 2 \\
\hline & 25 & 3.95 & 0 & 0 & 0 & 0 & 0 & 0 & 0 & 0 & 0 & 0 \\
\hline
\end{tabular}

- Abbreviations of the types of colonies are as described in Table I. 
These observations may suggest that, if there is a common lympho-hemopoietic origin, commitment to a lymphoid lineage may be later than CFU-GEMM. Sequential replating of hemopoieic blast cell colonies in a system permissive for both lymphoid and hemopoietic expression may clarify the origin of the lymphocytes detected in the hemopoietic colonies.

Stem cells must maintain their own population and provide cells for differentiation. 2 of the 30 human blast cell colonies formed secondary colonies that resembled the parent colony. All of the colonies produced secondary colonies, with GM the most frequently observed. The value of such an analysis depends upon the ability of the culture system to support the growth of these cells. Thus far, the incidence of multipotential progenitors in human blast cell colonies is lower than that we recently reported for mouse cells. Human hemopoietic progenitors have traditionally been more difficult to assay. In fact, we have failed to identify blast cell colonies in culture of adult marrow or peripheral blood cells. This may be due to lower incidences of progenitors for blast cell colonies. Alternatively, it may be reflecting technical limitations of the current assay system. During the long culture period for blast cells, the semi-solid medium can dehydrate and important nutrients and growth factors may decay or be consumed. Further improvement of the replating conditions is necessary for confirmation of the self-renewal capacity of human blast cell colonies.

\section{ACKNOWLEDGMENTS}

We would like to thank Mrs. Lobelia A. Avila, Mrs. Geneva Winkler, and Mrs. Karen L. Bergmann for their excellent technical assistance; Dr. Pamela N. Porter and Mrs. Anne G. Leary for their advice on the manuscript.

This work was supported by National Institutes of Health grants HL20913 and AM22170 and the Veterans Administration. Dr. Ogawa is a Veterans Administration Medical Investigator.

\section{REFERENCES}

1. Johnson, G. R., and D. Metcalf. 1977. Pure and mixed erythroid colony formation in vitro stimulated by spleen conditioned medium with no detectable erythropoietin. Proc. Natl. Acad. Sci. USA. 74: 3879-3882.

2. Hara, H., and M. Ogawa. 1978. Murine hemopoietic colonies in culture containing normoblasts, macrophages, and megakaryocytes. Am. J. Hematol. 4: 23-34.

3. Fauser, A. A., and H. A. Messner. 1979. Identification of magakaryocytes, macrophages, and eosinophils in colonies of human bone marrow containing neutrophilic granulocytes and erythroblasts. Blood. 53: 1023-1034.

4. Johnson, G. 1980 . Colony formation in agar by adult bone marrow multipotential hemopoietic cells. J. Cell. Physiol. 103: 371-383.

5. Humphries, R. K., A. C. Eaves, and C. J. Eaves. 1981. Self-renewal of hemopoietic stem cells during mixed colony formation in vitro. Proc. Natl. Acad. Sci. USA. 78: 3629-3633.

6. Ash, R. C., R. A. Detrick, and E. D. Zanjani. 1981. Studies of human pluripotential hemopoietic stem cells (CFU-GEMM) in vitro. Blood. 58: 309-316.

7. Nakahata, T., and M. Ogawa. 1982. Identification in culture of a class of hemopoietic colony-forming units with extensive capability to self-renew and generate multipotential colonies. Proc. Natl. Acad. Sci. USA. 79: 3843-3847.

8. Nakahata, T., S. S. Spicer, and M. Ogawa. 1982. Clonal origin of human erythro-eosinophilic colonies in culture. Blood. 59: 857-864.

9. Iscove, N. N., F. Sieber, and K. H. Winterhalter. 1974. Erythroid colony formation in cultures of mouse and human bone marrow. Analysis of the requirement for erythropoietin by gel filtration and affinity chromatography on agarose-concanavalin A. J. Cell. Physiol. 83: 309-320.

10. Messner, H. A., N. Jamal, and C. Izaguirre. 1982. The growth of large megakaryocyte colonies from human bone marrow. J. Cell. Physiol. (Suppl. 1): 45-51.

11. Nakahata, T., and M. Ogawa. 1982. Clonal origin of murine hemopoietic colonies with apparent restriction to granulocyte-macrophage-megakaryocyte (GMM) differentiation. J. Cell. Physiol. 111: 239-246.

12. Messner, H. A., C. A. Izaguirre, and N. Jamal. 1981. Identification of T-lymphocytes in human mixed hemopoietic colonies. Blood. 58: 402-405. 